\title{
Agreement of noninvasive tear break-up time measurement between Tomey RT-7000 Auto Refractor-Keratometer and Oculus Keratograph 5M
}

\author{
This article was published in the following Dove Press journal: \\ Clinical Ophthalmology \\ 16 September 2016 \\ Number of times this article has been viewed
}

\author{
Ryan Lee ${ }^{1,2}$ \\ Sharon Yeo' \\ Han Tun Aung ${ }^{3}$ \\ Louis Tong 1,2,4,5 \\ 'Ocular Surface Research Group, \\ Singapore Eye Research Institute, \\ ${ }^{2}$ Yong Loo Lin School of Medicine, \\ National University of Singapore, \\ ${ }^{3}$ School of Health Sciences, Ngee \\ Ann Polytechnic, ${ }^{4}$ Duke-NUS \\ Medical School, Singapore; ${ }^{5}$ Cornea \\ and External Eye Disease Service, \\ Singapore National Eye Centre, \\ Singapore
}

\begin{abstract}
Background: It is difficult to standardize assessment of dry eye in different clinical settings. Increasingly, tear stability is recognized to be important for the definition and assessment of patients with dry eye. Recently, two commercially available instruments have been made available for objectively measuring noninvasive tear break-up time (NIBUT), as an indicator of tear stability: the Tomey RT-7000 Auto Refractor-Keratometer and Oculus Keratograph (K)5M. We aim to assess the agreement of NIBUT measurements using these modalities.
\end{abstract}

Methods: This prospective cross-sectional study was carried out in a tertiary referral eye center and involved 126 consecutive dry eye patients. NIBUT assessment was performed on the right eyes of participants with both the RT-7000 and the K5M techniques, with the order of assessment randomized. The Standardized Patient Evaluation of Eye Dryness (SPEED) questionnaires were administered to assess dry eye symptoms in the 2 weeks before assessment.

Results: The age of the participants was $56.0 \pm 14.3$ years ( $69.84 \%$ females). Measurements for both modalities were non-normally distributed (right-skewed). The median RT-7000 and K5M readings were 4.2 (range $0.1-10.0$ ) and $6.4(0.1-24.9)$ seconds, respectively. RT-7000 and K5M readings were poorly correlated $(\rho=0.061, P=0.495)$. Intraclass correlation coefficient between the modalities was 0.187 ( $95 \%$ confidence interval -0.097 to 0.406 ). The Bland-Altman plot showed no systematic differences between the readings with these machines. The agreement between machines was not different in different SPEED categories.

Conclusion: While there are theoretical and practical benefits of NIBUT for assessment of tear stability over dye-based methods, the agreement between the two modalities was poor. Hence, studies and trials assessing NIBUT should avoid using these modalities interchangeably for NIBUT assessment. More research is needed to improve consensus on how to determine NIBUT.

Keywords: tear break-up time, tear film, Oculus Keratograph, Tomey RT-7000, dry eye

\section{Introduction}

Dry eye syndrome is a multifactorial disorder of the ocular surface with significant health care burden, causing ocular discomfort, visual disturbance, and tear film instability with potential damage to the ocular surface. ${ }^{1,2}$ The tear film nourishes the avascular cornea and also contributes to the refractive power of the eye. ${ }^{3,4}$ An unstable tear film that breaks down rapidly in between blinks results in excessive exposure of the cornea, leading to devitalized corneal epithelium and visual disturbances. ${ }^{4}$ Tear film instability is hence a key feature of dry eye.
Correspondence: Louis Tong Cornea and External Eye Disease Service, Singapore National Eye Center, II Third Hospital Avenue, Singapore 16875 I

Tel +65 62277255

Fax +65 63224599

Email louis.tong.h.t@snec.com.sg 
The most widely used clinical test for assessing tear instability is the measurement of fluorescein tear break-up time (FTBUT) ${ }^{5,6}$ Unfortunately, despite its widespread use in both clinical and research settings, it is recognized that FTBUT has poor accuracy and reproducibility. ${ }^{7}$ A few characteristics of the test contribute to its poor performance. First, the variability in the volume of fluorescein instilled into the conjunctival fornices is likely to be a major contributor to the poor performance of the test. ${ }^{8,9}$ Moistened fluorescein strips deliver an inconsistent volume of fluorescein to the ocular surface, which, in turn, directly affects the FTBUT measurement obtained. ${ }^{9}$ This is especially the case because the original tear volume is a minute volume, and addition of even a tiny amount of fluorescein solution significantly disturbs native tear dynamics. This may explain why studies that use customized fluorescein strips, which deliver smaller volumes of fluorescein, obtain more repeatable results. ${ }^{10,11}$ A second source of error is the observer. The ability to detect the end point of the test (observing tear break-up) is affected by the brightness of the cobalt-blue-filtered light used and the individual assessor's proficiency with slit-lamp examination. ${ }^{12}$ Overall, the difficulty in standardizing these factors has limited the repeatability of FTBUT measurements. ${ }^{13}$

The development of techniques that measure noninvasive tear break-up time (NIBUT) addresses many of these inherent limitations associated with FTBUT. Earlier devices such as tearscope allowed visualization of the tear film without fluorescein, hence enabling assessment of the tear film in its undisturbed state. ${ }^{14}$ More recent techniques employ predefined computer algorithms for the determination of tear break-up time, ensuring consistent detection of tear film break-up in an objective and automated manner. ${ }^{15,16}$

While many prototypes have been developed to measure NIBUT, there are currently only two commercially available systems: the Tomey RT-7000 Auto Refractor-Keratometer (Tomey Corporation, Nagoya, Japan) and the Oculus Keratograph 5M (Oculus, Wetzlar, Germany). Previous reports have focused on the agreement of NIBUT with traditional FTBUT, or the difference in NIBUT between dry eye patients and healthy volunteers. ${ }^{15-17}$ While each individual system shows good performance in differentiating dry eye patients from healthy controls, they each employ different methodologies to monitor the tear film and detect tear break-up. There has not been any published study that compared these two modalities. In this study, we examined the intraobserver agreement of NIBUT readings obtained by the previously mentioned modalities in a relatively large hospital-based sample of dry eye patients.

\section{Methodology}

This study was approved by the Singapore Health Services Centralized Institutional Review Board. It adhered to the tenets of the Declaration of Helsinki for clinical research, and written informed consent was obtained from all the participants after explanation of the purpose and possible consequences of the study.

This prospective observational study was carried out in the Singapore National Eye Center, a tertiary referral center. All consenting participants with a previous diagnosis of dry eye were considered eligible for the study. A total of 126 consecutive participants were recruited from the dry eye clinic and underwent standardized assessments.

Each participant underwent NIBUT assessment with both the Tomey RT-7000 Auto Refractor-Keratometer RT-7000 and the Oculus Keratograph (K) 5M, one reading was obtained for each instrument with 10 minutes interval between measurements to ensure that normal tear function was restored. The order of assessment was randomized, and only the right eye of each participant was examined.

The RT-7000 measures NIBUT by assessing the reflectivity of the tear film. As the patient was instructed to refrain from blinking and to fixate on a central light source, 15 mire rings from a lighted cone were projected onto the corneal surface. The native software captures images of the reflected rings at each second up to a 10 -second duration, starting from the last blink. At each given second, the computer compares the brightness of the reflected rings with the initial brightness at zero seconds. The software takes into account 256 measurement points per mire ring, resulting in a total of 3,840 measurement points. If the brightness falls below a predefined threshold, the computer records the point at which the threshold is crossed as the tear break-up time, which is termed ring break-up time (RBUT).

The K5M measures NIBUT by detecting localized breaks in the tear film using infrared waves. After two blinks to reconstitute the tear film, the participant is instructed to refrain from blinking and fixate on the central light source. A video recording of the ocular surface begins, with real-time detection and localization of breaks in the tear film. During the assessment, 22 rings are projected onto the cornea, with more than 1,000 measurement points per ring, resulting in 22,000 analyzed data points per frame. Points of break-ups appear on a grid mapping the corneal surface. The video recording lasts up to a maximum of 25 seconds, or until the patient's next blink, whichever occurs first. Two readings are provided at the end of every assessment: NIKBUT-First, the time taken for the first appearance of a break in the tear 
film, is the parameter of interest in this study. The other reading, produced by $\mathrm{K} 5 \mathrm{M}$, called the NIKBUT-Average is the average of the time taken to break-up in all the regions monitored over the duration of the 25 seconds. The NIKBUTAverage is therefore higher than the NIKBUT-First, and only the latter will be analyzed in this report.

Each participant also underwent the validated Standard Patient Evaluation of Eye Dryness (SPEED) questionnaire for assessment of frequency and severity of dry eye symptoms. ${ }^{18}$ Four common symptoms of dry eye were assessed, namely, dryness, soreness, burning sensation, and eye fatigue. Frequency of each symptom was scored from 0 to 3 , with 0 being "never" and 3 being "all the time". Intensity of each symptom was scored from 0 to 4 , with 0 being "not a problem" and 4 being "intolerable". These symptoms were asked for the prior 2 weeks' duration. These scores were added to give a total score (0-28), with a higher score indicating increasing more severe dry eye symptoms. History of any ocular surgery such as cataract removal or laser refractive surgery was also recorded.

Statistical analysis was performed with SPSS version 20 (IBM Corporation, Armonk, NY, USA). Descriptive statistics were calculated, including scatter plots. Normality for variables was checked using the Kolmogorov-Smirnov test. Correlation between RT-7000 and K5M measurements was performed using Spearman's rho correlation. Bland-Altman plot was constructed to assess agreement between the two modalities. Intraclass correlation coefficient for agreement was calculated using a two-way random model for absolute agreement. Level of significance was set at $P=0.05$.

\section{Results}

The demographic characteristics of the 126 participants are presented in Table 1. The majority of our participants were of Chinese ethnicity. The dry eye symptoms were mild to moderate in this study. Within this range, the correlation between NIBUT, as measured by both instruments, and SPEED was poor $(\rho=0.0003, P=0.997$ and $\rho=0.085, P=0.344$ for RT-7000 and K5M, respectively).

The NIBUT readings obtained for both RT-7000 and K5M were non-normally distributed (Figure $1 \mathrm{~A}$ and $\mathrm{B}$ ). For RT-7000, 28.6\% of cases had NIBUT of 10 seconds, which was the maximum duration recorded by the machine. Using K5M, very few (3.2\%) achieved the maximum reading of 25 seconds. Omitting the maximal NIBUT, the distribution of the RT-7000 and K5M readings were rightskewed and non-normally distributed (Kolmogorov-Smirnov test, $P<0.001)$.
Table I Demographic characteristics and parameters of interest $(n=126)$

\begin{tabular}{ll}
\hline Age, years & $56.04(\mathrm{I} 4.32)$ \\
Sex, female & $88(69.84)$ \\
Ethnicity & \\
$\quad$ Chinese & $122(96.83)$ \\
$\quad$ Malay & $2(1.59)$ \\
$\quad$ Indian & $2(1.59)$ \\
Tomey RT-7000 NIBUT, seconds & \\
$\quad$ Mean (SD) & $4.95(3.95)$ \\
$\quad$ Median (range) & $4.15(0.1-10.0)$ \\
Oculus K5M NIBUT, seconds & \\
$\quad$ Mean (SD) & $8.39(6.00)$ \\
$\quad$ Median (range) & $6.35(0.1-24.9)$ \\
SPEED questionnaire score & \\
$\quad$ Mean (SD) & $8.84(5.81)$ \\
Median (range) & $8.00(0.0-28.0)$ \\
\hline
\end{tabular}

Notes: Values are presented as mean (SD) or median (range) with exception of sex and ethnicity, which are in number (percentages).

Abbreviations: NIBUT, noninvasive tear break-up time; SPEED, Standard Patient Evaluation of Eye Dryness; SD, standard deviation.

We examined the scatter plots for the measurements (Figure 2) and when linear correlation was calculated with or without the 10 -second RT-7000 readings (28\% of total readings), the correlation was poor, with $\rho=0.061(P=0.495)$ and $0.023(P=0.826)$, respectively. We also examined the correlation between the two techniques separately for cases that had K5M performed before RT-7000 and those that had the other sequence. There was no obvious difference in correlation when K5M was performed first or after RT-7000 ( $\rho=0.31, P=0.25$ when $\mathrm{K} 5 \mathrm{M}$ was performed first; $\rho=0.087$, $P=0.77$ when RT-7000 was performed first).

Intraclass correlation coefficient for agreement between RT-7000 and K5M for average measures was 0.187 (95\% confidence interval [CI] -0.097-0.406). Bland-Altman plot (Figure 3) of the NIBUT values shows poor agreement between the RT-7000 and K5M techniques. In particular, the extent of agreement was not related to the magnitude of the readings, ie, lack of agreement was not greater in larger readings. There was also no systematic difference between the readings obtained from the two techniques.

\section{Discussion}

In this study, we examined the agreement between the two commercially available modalities for measuring NIBUT. Perhaps, because of the vastly different algorithms used for measuring NIBUT, these two modalities produced low agreement which was clinically relevant.

Till date, no published studies have directly compared NIBUT assessment by these two techniques. Nevertheless, Izquierdo et al compared K5M NIBUT with FTBUT 

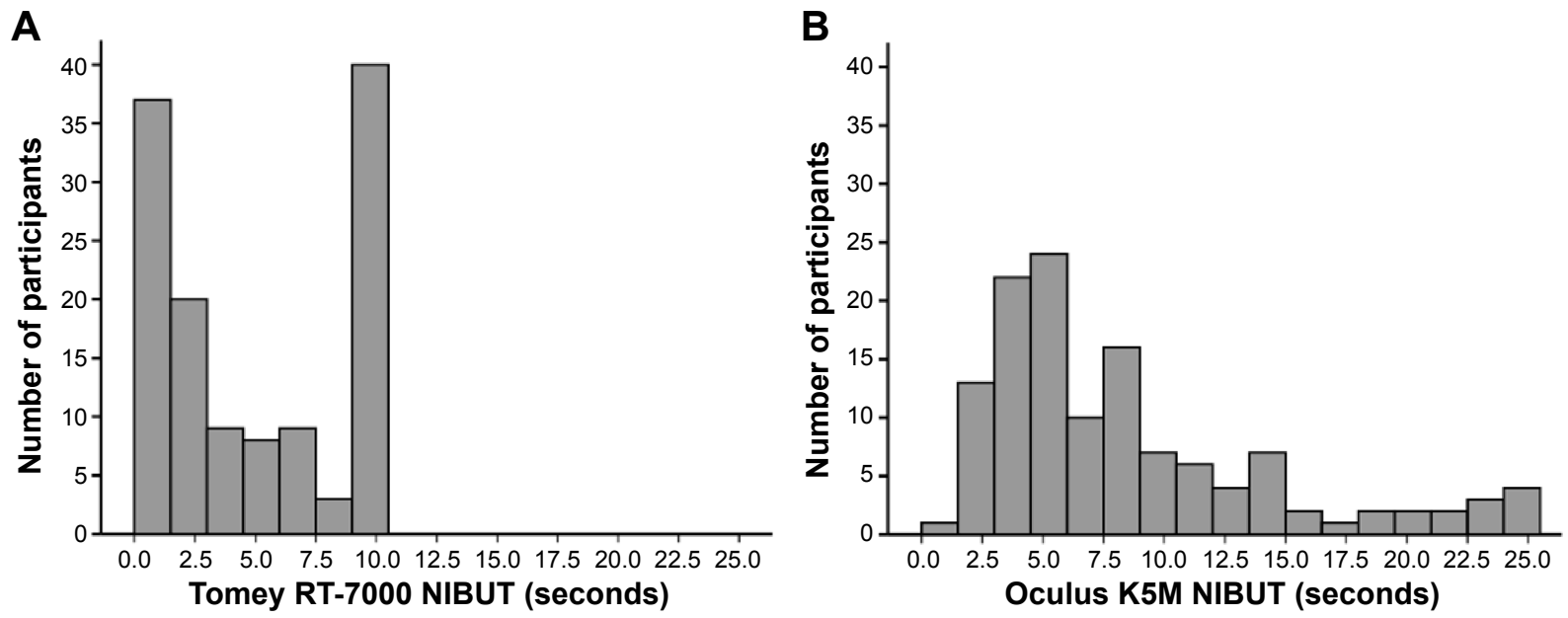

Figure I Histograms showing distribution of noninvasive break-up time readings of the I 26 participants in the study, in Tomey RT-7000 (A) and Oculus Keratograph 5 M (B). Abbreviation: NIBUT, noninvasive tear break-up time.

and found that there was moderate correlation $(r=0.68) .{ }^{17}$ Cox et al examined an earlier model of K5M (Oculus Keratograph 4) for its agreement with FTBUT and inter-visit repeatability of Keratograph 4-determined NIBUT, with similar findings that agreement was moderate. ${ }^{19}$ Best et al found that the $\mathrm{K} 5 \mathrm{M}$ readings are consistently shorter than those determined by tearscope. ${ }^{20}$ In contrast, the repeatability of RT-7000-determined NIBUT and agreement of RT-7000 with FTBUT/fearscope has not been published. A few studies have assessed NIBUT as a potential diagnostic marker for dry eye. Gumus et al performed RT-7000 measurements on 45 dry eye patients and 25 healthy volunteers. The RT-7000 readings decreased with increasing severity of dry eye (modified Dry Eye Workshop scale ${ }^{21}$ ). In controls and more severe dry eye patients, mean NIBUT was $4.91 \pm 1.62$ and $0.36 \pm 0.45$ seconds, respectively. Using a diagnostic threshold of $<5$ seconds, sensitivity and specificity of RT-7000 for

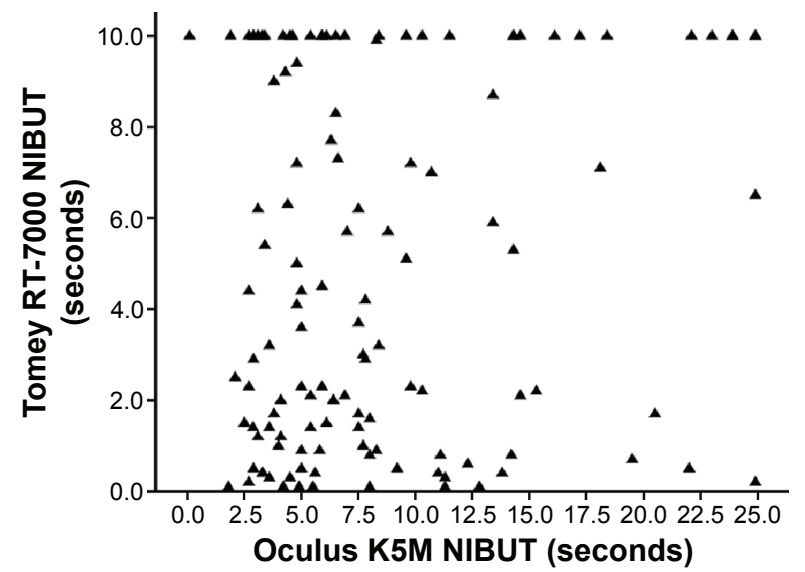

Figure 2 Scatter plot showing correlation between noninvasive break-up time readings of Tomey RT-7000 and Oculus Keratograph 5M.

Abbreviation: NIBUT, noninvasive tear break-up time. diagnosing dry eye were $82 \%$ and $60 \%$, respectively. ${ }^{16}$ Abdelfattah et al performed a similar study with K5M with many more participants (total 159). ${ }^{15}$ This study found no significant difference $(P=0.69)$ in NIBUT between dry eye participants and healthy volunteers (mean of 6.7 and 8.2 seconds, respectively). It is notable that the diagnosis of dry eye was based on individual clinician's judgment, but the exact diagnostic criteria for such diagnoses were not specifically reported. Since tear instability may contribute to different extents in patients from separate settings, these studies may not be directly comparable.

The two techniques, though both apply Placido-ring strategies, use vastly different algorithms and thresholds for measurement of NIBUT. The RT-7000 measures overall reflectivity of the entire tear film by assessing the brightness of the reflection it produces. Only one image is taken per second, and each image has a total of 3,840 measurement

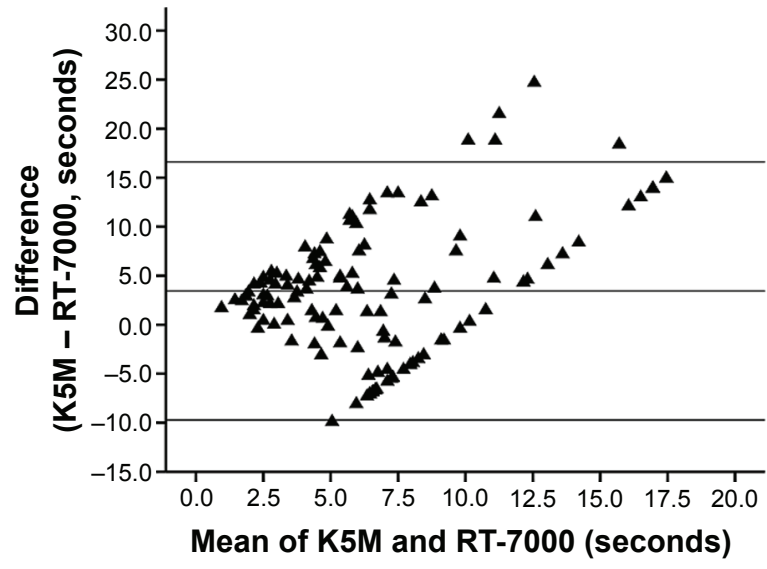

Figure 3 Bland-Altman plot of noninvasive break-up time readings of Tomey RT-7000 and Oculus Keratograph 5M. 
points distributed over 15 mire rings. A parameter called the "ring break unit" is calculated as the reflection brightness decreases, and when the threshold of 20 is reached, the break-up time is recorded. This algorithm may be good for assessing global changes but does not discriminate differences in the spatial locations of tear break-ups. The $\mathrm{K} 5 \mathrm{M}$ tracks tear film integrity through infrared waves and captures images at 32 frames per second, which is suitable for measuring rapid changes. The resolution of each image produced by K5M (22,000 measurement points) is also much higher than that produced by RT-7000. Unlike the single reading of NIBUT in RT-7000, K5M produced two measurements per eye, the NIKBUT-First and the NIKBUT-Average.

The strength of this study is a consecutively recruited relatively large sample. Measurement of the same eye with different instruments was performed in a randomized order with equal frequency. The limitations include the lack of assessment of clinical signs associated with dry eye, such as corneal fluorescein staining and Schirmer's test. However, the aim of this paper was not to examine the usefulness of these two instruments for detection of dry eye.

Based on the benefits of NIBUT discussed in the "Introduction" section, it is a useful parameter for assessing tear stability. However, poor agreement between the readings from these two machines suggests that in clinical trials, the same modality should be used in the repeated measurements of participants. There is insufficient evidence at this point to conclude if one modality is superior to the other. In general, a wider range of measurements may be preferable to a narrow range. In the case of RT-7000, restricting the measurement time to a cap of 10 seconds may limit its usefulness.

For NIBUT to be more widely acceptable to clinicians, it is important for commercial developers to reveal underlying algorithms to allow for further analysis. For instance, greater definition of imaging (more frames per second) is good for tracking the millisecond to millisecond changes in the tear film. Greater resolution of the individual frames will enable the modality to be more sensitive to even the tiniest breaks in the tear film. The method of patient fixation and position should also be assessed or measured. Each of these factors influences the performance of NIBUT measuring techniques and should be made available, so that clinicians can understand the individual strengths and weaknesses of each modality. Future developments may include detection of patterns of break-up (eg, spot, area, and random breaks) to delineate specific pathology such as poor wettability, etc.

\section{Conclusion}

The two commercially available modalities for NIBUT assessment have poor clinical agreement with one another. While NIBUT is potentially very useful, more research into defining NIBUT is needed for determining the appropriate algorithm for routine clinical use.

\section{Acknowledgments}

We would like to sincerely thank Too Cheah Loon, Cynthia Boo, Loh Wen Xin, Chan Shi Kei, Marc Ng Yen Xing, Fong Jian Ming, Toh Li Xin, Ng Qiu Mei, and Trina Ho Yi En for aiding us with the recruitment, data collection, and data management of this study.

\section{Disclosure}

The authors report no conflicts of interest in this work.

\section{References}

1. The definition and classification of dry eye disease: report of the Definition and Classification Subcommittee of the International Dry Eye WorkShop (2007). Ocul Surf. 2007;5(2):75-92.

2. Yu J, Asche CV, Fairchild CJ. The economic burden of dry eye disease in the United States: a decision tree analysis. Cornea. 2011;30(4): 379-387.

3. Korb DR. The tear film: its role today and in the future. In: Korb DR, Craig J, Doughty M, Guillion J-P, Tomlinson A, Smith G, editors. The Tear Film: Structure, Function and Clinical Examination. Boston: Butterworth-Heinemann; 2002:9-13.

4. Ousler GW 3rd, Hagberg KW, Schindelar M, Welch D, Abelson MB The ocular protection index. Cornea. 2008;27(5):509-513.

5. Norn MS. Desiccation of the precorneal film. I. Corneal wetting-time. Acta Ophthalmol. 1969;47(4):865-880.

6. Lemp MA. Breakup of the tear film. Int Ophthalmol Clin. 1973;13(1): 97-102.

7. Vanley GT, Leopold IH, Gregg TH. Interpretation of tear film breakup. Arch Ophthalmol. 1977;95(3):445-448.

8. Foulks GN. Challenges and pitfalls in clinical trials of treatments for dry eye. Ocul Surf. 2003;1(1):20-30.

9. Johnson ME, Murphy PJ. The Effect of instilled fluorescein solution volume on the values and repeatability of TBUT measurements. Cornea. 2005;24(7):811-817.

10. Korb DR, Greiner JV, Herman J. Comparison of fluorescein break-up time measurement reproducibility using standard fluorescein strips versus the dry eye test (DET) method. Cornea. 2001;20(8):811-815.

11. Pult H, Riede-Pult BH. A new modified fluorescein strip: its repeatability and usefulness in tear film break-up time analysis. Cont Lens Anterior Eye. 2012;35(1):35-38.

12. Cho P, Brown B, Chan I, Conway R, Yap M. Reliability of the tear break-up time technique of assessing tear stability and the locations of the tear break-up in Hong Kong Chinese. Optom Vis Sci. 1992;69(11): 879-885.

13. Savini G, Prabhawasat P, Kojima T, Grueterich M, Espana E, Goto E. The challenge of dry eye diagnosis. Clin Ophthalmol. 2008;2(1): 31-55.

14. Maissa C, Guillon M. Tear film dynamics and lipid layer characteristics effect of age and gender. Cont Lens Anterior Eye. 2010;33(4):176-182.

15. Abdelfattah NS, Dastiridou A, Sadda SR, Lee OL. Noninvasive Imaging of tear film dynamics in eyes with ocular surface disease. Cornea. 2015; 34(Suppl 10):S48-S52. 
16. Gumus K, Crockett $\mathrm{CH}$, Rao K, et al. Noninvasive assessment of tear stability with the tear stability analysis system in tear dysfunction patients. Invest Ophthalmol Vis Sci. 2011;52(1):456-461.

17. Izquierdo L, Henriquez MA, Rodriguez M, Rodriguez L. Clinical versus automatic keratographer evaluation for the diagnosis of dry eye syndrome. Invest Ophthalmol Vis Sci. 2014;55(13).

18. Ngo W, Situ P, Keir N, Korb D, Blackie C, Simpson T. Psychometric properties and validation of the Standard Patient Evaluation of Eye Dryness questionnaire. Cornea. 2013;32(9):1204-1210.
19. Cox SM, Nichols KK, Nichols JJ. Agreement between automated and traditional measures of tear film breakup. Optom Vis Sci. 2015;92(9): e257-e263.

20. Best N, Drury L, Wolffsohn JS. Clinical evaluation of the Oculus Keratograph. Cont Lens Anterior Eye. 2012;35(4):171-174.

21. Sullivan BD, Whitmer D, Nichols KK, et al. An objective approach to dry eye disease severity. Invest Ophthalmol Vis Sci. 2010;51(12): 6125-6130.

\section{Publish your work in this journal}

Clinical Ophthalmology is an international, peer-reviewed journal covering all subspecialties within ophthalmology. Key topics include: Optometry; Visual science; Pharmacology and drug therapy in eye diseases; Basic Sciences; Primary and Secondary eye care; Patient Safety and Quality of Care Improvements. This journal is indexed on

\section{Dovepress}

PubMed Central and CAS, and is the official journal of The Society of Clinical Ophthalmology (SCO). The manuscript management system is completely online and includes a very quick and fair peer-review system, which is all easy to use. Visit http://www.dovepress.com/ testimonials.php to read real quotes from published authors. 